\title{
A BeppoSAX observation of Her X-1 during the first main-on after an anomalous low-state: Evidence for rapid spin-down
}

\author{
T. Oosterbroek ${ }^{1}$, A. N. Parmar ${ }^{1}$, M. Orlandini ${ }^{2}$, A. Segreto ${ }^{3}$, A. Santangelo ${ }^{3}$, and S. Del Sordo ${ }^{3}$ \\ 1 Astrophysics Division, Space Science Department of ESA, ESTEC, PO Box 299, 2200 AG Noordwijk, \\ The Netherlands \\ 2 Istituto TESRE, CNR, via Gobetti 101, 40129 Bologna, Italy \\ 3 IFCAI, CNR, via La Malfa 153, 90146 Palermo, Italy
}

Received 17 April 2001 / Accepted 5 June 2001

\begin{abstract}
Results of a BeppoSAX observation of Her X-1 in 2000 October during the first main-on state after the longest recorded anomalous low-state are presented. The $0.1-30 \mathrm{keV}$ spectrum, light curve and pulse profile are all consistent with those measured during previous main on-states, indicating that Her X-1 has resumed its regular 35 day cycle with similar on-state properties as before. However, from a comparison of the measured pulse period with that obtained close to the start of the anomalous low-state, it is evident that Her X-1 continued to spin-down strongly during the anomalous low-state such that the pulse period has returned to a similar value as $\sim 15$ years ago. Additionally, the occurrence time of the main-on states after the end of the anomalous low-state indicate that a change in the length, or phasing, of the 35-day cycle occurred during the anomalous low-state.
\end{abstract}

Key words. accretion, accretion disks - X-rays: binaries - stars individual: Her X-1

\section{Introduction}

Her X-1 is an eclipsing binary X-ray pulsar with a pulse period of $1.24 \mathrm{~s}$ and an orbital period of 1.7 days (Tananbaum et al. 1972; Giacconi et al. 1973). Normally, the source exhibits a 35 day X-ray intensity cycle comprising a $\sim 10$ day duration main on-state and a fainter $\sim 5$ day duration secondary on-state approximately half a cycle later. At other phases of the 35 day cycle, Her X-1 is still visible at a low level (Jones \& Forman 1976). This modulation has been ascribed to a tilted precessing accretion disk that periodically obscures the line of sight to the neutron star (Gerend \& Boynton 1976). In addition, a regular pattern of X-ray intensity dips is usually observed at certain orbital phases. These may be caused by obscuration from periodically released matter from the companion star (Crosa \& Boynton 1980).

The 35-day cycle has been evident in RXTE All-Sky Monitor (ASM) $1.5-12 \mathrm{keV}$ data with the main-on state being clearly detected every 35 days for $>3$ years (e.g., Scott \& Leahy 1999). An exception to this regularity occurred when the on-state expected around 1999 March 23 was not detected (Levine \& Corbet 1999). Observations with BeppoSAX showed only low level activity during the expected time of the next main-on (Parmar et al. 1999).

Send offprint requests to: T. Oosterbroek, e-mail: toosterb@astro.estec.esa.nl
Similar exceptions have been detected twice before. In 1983 June to August EXOSAT failed to detect an X-ray on-state from Her X-1 and instead a faint source, with a strength comparable to that of the low-state emission was observed over a wide range of 35-day phases (Parmar et al. 1985). Optical observations during this interval, and in 1999 April, detected the effects of strong X-ray heating on the companion star (HZ Her) indicating that it was still being irradiated by a strong X-ray source (Delgado et al. 1983; Margon et al. 1999). The 1983 anomalous lowstate lasted $<0.8$ year with the 35 -day cycle returning by 1984 March. Similarly, in 1993 August ASCA failed to observe the expected on-state, again detecting instead a faint X-ray source (Vrtilek et al. 1994; Mihara \& Soong 1994). The latest anomalous low-state is the longest ever observed, lasting 1.5 years. During the beginning of this anomalous low-state a rapid spin-down of the pulse period was observed (Parmar et al. 1999; Coburn et al. 2000), which was interpreted as resulting from a torque-reversal, possibly caused by a reversal of the rotation of the inner disk (see e.g., van Kerkwijk et al. 1998).

Here we report on a BeppoSAX observation during the first main-on state (as derived from the RXTE-ASM light curve) after the 1999 March to 2000 September anomalous low-state had ended. We discuss the source properties and show that the $0.1-30 \mathrm{keV}$ spectrum, light curves and pulse profiles are indistinguishable from those during previous 


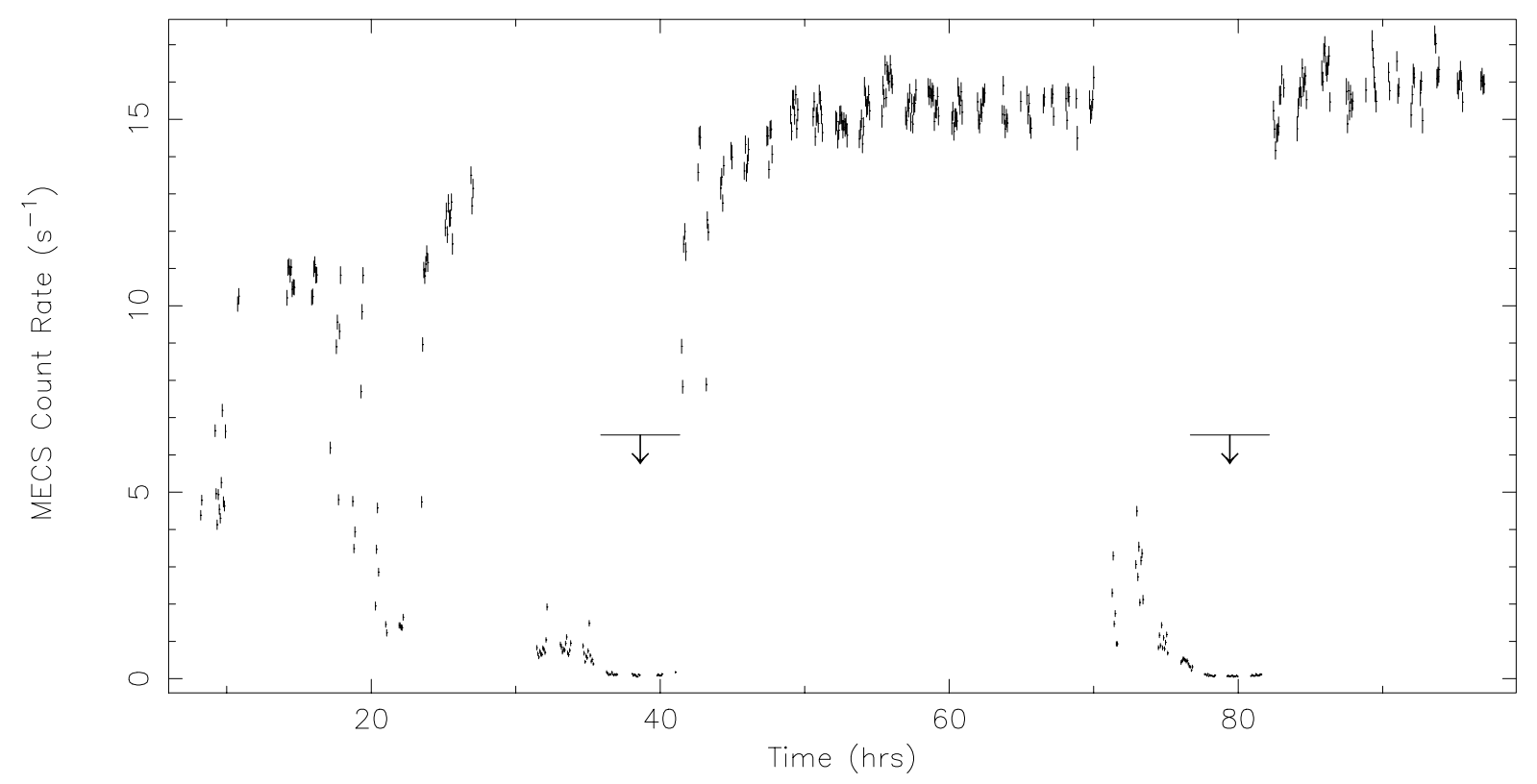

Fig. 1. The MECS $2-10 \mathrm{keV}$ light curve of Her X-1 with a binning of $256 \mathrm{~s}$. The time is hours of 2000 October 9 . The eclipse intervals are indicated by arrows and horizontal lines.

high-states, but that the pulse period probably continued its strong spin-down behavior during the anomalous lowstate itself. Additionally, we examine the 35 day turn-on times after and before the anomalous low-state and find that the 35 day period, or phasing, changed during the anomalous low-state.

\section{Observations}

Results from the Low-Energy Concentrator Spectrometer (LECS; 0.1-10 keV; Parmar et al. 1997), the MediumEnergy Concentrator Spectrometer (MECS; 1.8-10 keV; Boella et al. 1997), and the Phoswich Detection System (PDS; 15-300 keV; Frontera et al. 1997) on-board BeppoSAX are presented. The MECS consists of two grazing incidence telescopes with imaging gas scintillation proportional counters in their focal planes. The LECS uses an identical concentrator system as the MECS, but utilizes an ultra-thin entrance window and a driftless configuration to extend the low-energy response to $0.1 \mathrm{keV}$. The non-imaging PDS consists of four independent units arranged in pairs each having a separate collimator. Each collimator was alternatively rocked on- and $210^{\prime}$ off-source every $96 \mathrm{~s}$ during the observation. The HPGSPC was not operated during this observation.

The observation was performed during the rising edge of the main-on between 2000 October 9 08:12 and October 13 01:08 (UTC). Examination of the RXTE-ASM light curve shows that the start of the on-state occurred a few hours earlier. This is also seen in the MECS light curve (Fig. 1) where the count rate at the start is already increasing and higher than the low-state value of $\sim 1 \mathrm{~s}^{-1}$ (see Oosterbroek et al. 2000). The standard LECS and MECS extraction radii of $8^{\prime}$ and $4^{\prime}$ were used. Standard data selection and background subtraction procedures were applied using the using the SAXDAS 2.0.0 data analysis package. The resulting exposures in the LECS, MECS, and PDS instruments are $28.5 \mathrm{ks}, 106.0 \mathrm{ks}$, and $48.1 \mathrm{ks}$, respectively.

\section{Analysis}

The pulse period of Her X-1 was determined. First, the arrival times of the photons were corrected to the solar system barycenter. Then, the arrival times were additionally corrected to the Her X-1 center of mass using the ephemeris of Deeter et al. (1991). The period was obtained with an epoch-folding technique using the MECS data, while the $(1 \sigma)$ uncertainties were determined by fitting the arrival times of 17 averaged profiles. We find a period of $1.2377697(3) \mathrm{s}$. This pulse period is plotted in Fig. 2 together with a compilation of previously obtained pulse periods (see Parmar et al. 1999; Coburn et al. 2000 for references).

The LECS and MECS spectra were rebinned to have $>20$ counts in each bin. Additionally, a rebinning was performed such that the full width half-maximum energy resolution is oversampled by at most a factor 3 to ensure the applicability of the $\chi^{2}$ statistic. A $1 \%$ systematic uncertainty was added to the rebinned data. Spectra were obtained during the whole of the second and third orbits (excluding dips and eclipses) and during 5 small intervals of the first orbit (since rapid variations are present). The LECS, MECS and PDS spectra were fit with the model described in Oosterbroek et al. (2000), which consists of a soft blackbody and a hard power-law, together with two Gaussian emission features near 6.5 and $1.0 \mathrm{keV}$ (describing two iron lines). The spectra obtained during the second and third orbits (see Fig. 3) are in all respects similar to the spectra obtained during previous main-on states, 


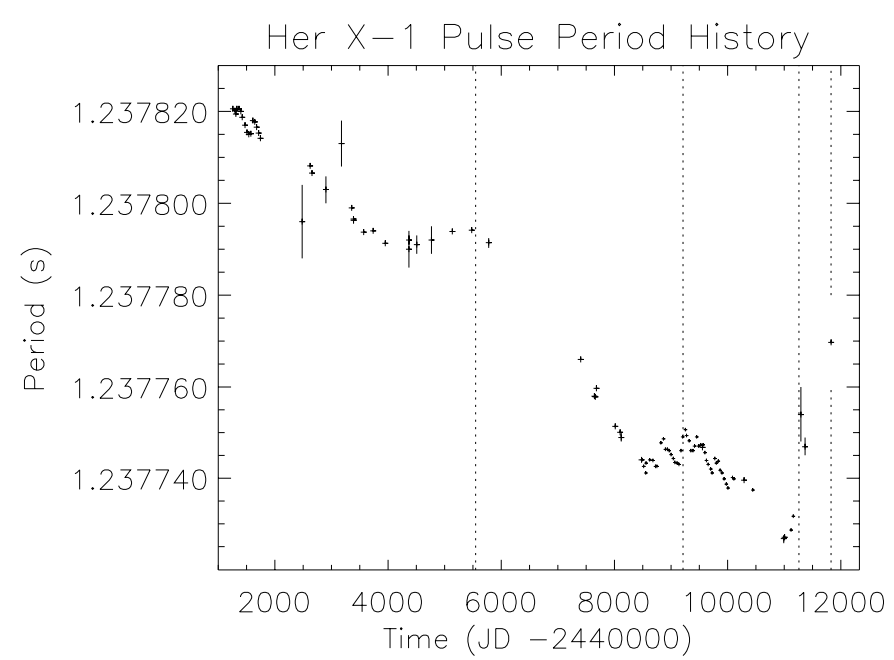

Fig. 2. The Her X-1 pulse period history. The two leftmost dashed vertical lines mark the previous anomalous low-states, while the two rightmost dashed vertical lines indicate the beginning and end of the latest anomalous low-state.

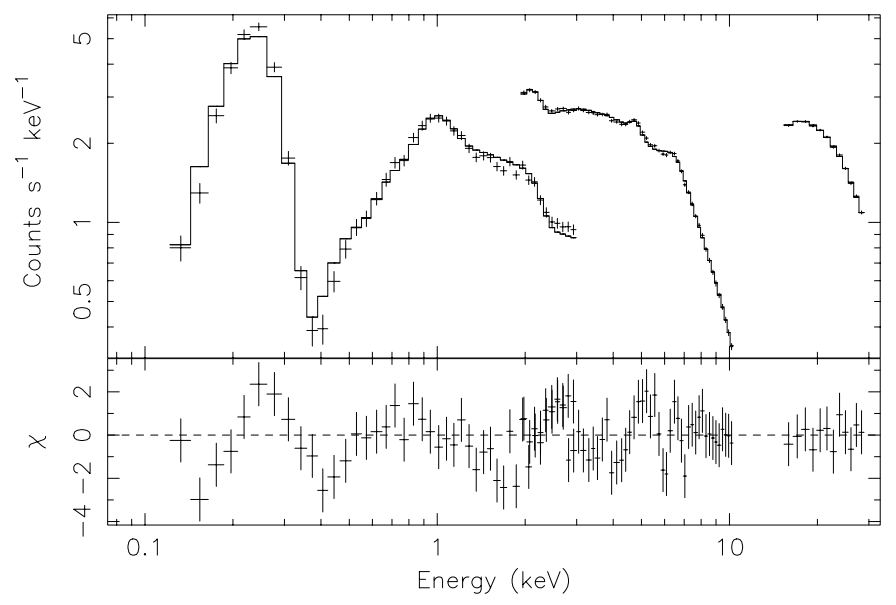

Fig. 3. The LECS, MECS and PDS count spectra obtained during the third orbit covering the $0.1-30 \mathrm{keV}$ energy range. The structured low-energy residuals are caused by the unusually low LECS detector temperature which is not properly modeled by the response generating software.

while the spectrum obtained during the first orbit can be adequately described with the standard model modified by partial covering. The spectrum (Fig. 3) was fit using the model in Dal Fiume et al. (1998), without the addition of the cyclotron line (since we limit our spectrum to $30 \mathrm{keV}$ ). This model consists of a broken powerlaw (with a break-energy $E_{\text {break }}$, and two power-law indices, $\alpha_{1}$ and $\alpha_{2}$ ) modified by a high-energy cutoff (characterized by $E_{\text {cutoff }}$, and, $\left.E_{\text {fold }}\right)$ two Gaussians, and a (soft) blackbody. The main parameters of the continuum are (with the Dal Fiume 1998 model 1 values in parentheses): $\alpha_{1} 0.89 \pm$ $0.01(0.884 \pm 0.003), E_{\text {break }}(\mathrm{keV}) 17.1 \pm_{0.8}^{0.3}\left(17.74 \pm_{0.30}^{0.26}\right)$, $\alpha_{2} 1.86 \pm 0.4\left(1.83 \pm_{0.07}^{0.05}\right), E_{\text {cutoff }}(\mathrm{keV}) 23.5 \pm 2(24.2 \pm 0.2)$, $E_{\text {fold }}(\mathrm{keV}) 15.5 \pm 5(14.8 \pm 0.4)$.

Therefore, the spectral parameters are completely consistent with those of the normal main-on spectrum, and we

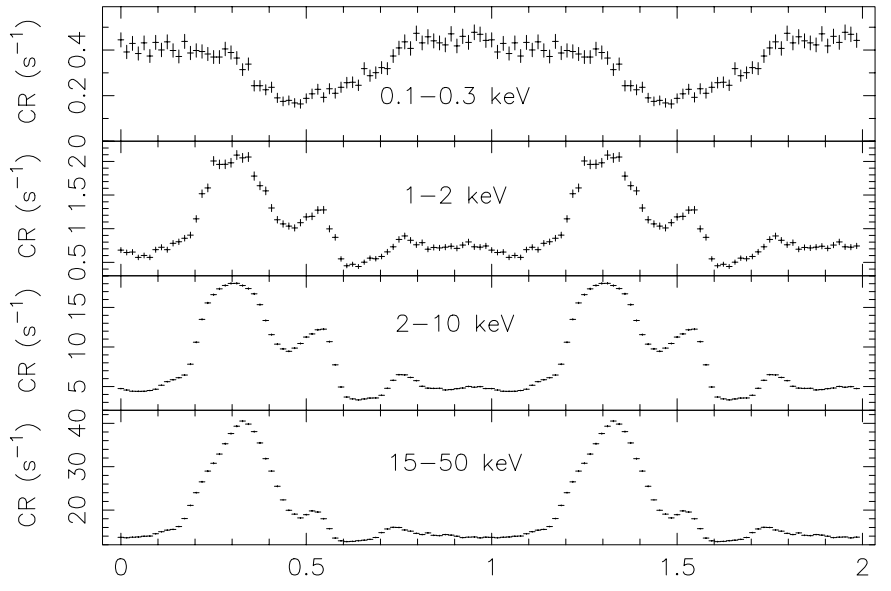

Fig. 4. The Her X-1 pulse profile in 4 energy bands (indicated in the panels). Pulse phase is arbitrary but is the same for each panel. From top to bottom the data are obtained with the LECS, LECS, MECS and PDS instruments. The PDS data have not been background subtracted.

therefore conclude that the $0.1-30 \mathrm{keV}$ spectrum during this main-on state is in no way remarkable.

In Fig. 4 the pulse profiles obtained in 4 different energy bands are plotted. These clearly show the well known transition from a broad, approximately sinusoidal, profile at low energies (associated with the soft blackbody component, see e.g., Oosterbroek et al. 1997) to the sharper double-peaked profile at higher energies (associated with the power-law component). They are very similar to e.g., the main-on observation reported in Oosterbroek et al. (1997).

\subsection{5 day turn-on time}

In order to investigate whether the 35 day on-states observed after the anomalous low-state occurred at times consistent with their ephemeris before the low-state RXTE-ASM data covering the interval 1996 January 5 to 2001 February 26 were used. Only ASM detections with a signal to noise ratio of $>7.5$ were included (this had the side-effect of excluding all but one measurement outside the main-on state - which was manually deleted). The mean occurrence time of each the main-on state intensity was determined to be the mean of the $2-12 \mathrm{keV}$ data points belonging to each main-on, while the uncertainty was taken as the the square root of the variance divided by the number of points (i.e. the error in the mean). We note that this is only a very rough estimate of the uncertainty, and does not take into account any systematic uncertainties. However, our results are insensitive to the choice of uncertainties. Whilst this is a very rough method, the results agree surprisingly well with the more detailed analysis (basically a template fitting method) of Shakura et al. (1998). Next a linear ephemeris was fit to the times of the main-ons that occurred before the anomalous lowstate to give a mean 35 day cycle time of 34.8 day, consistent with the model of Staubert et al. (1983) where 


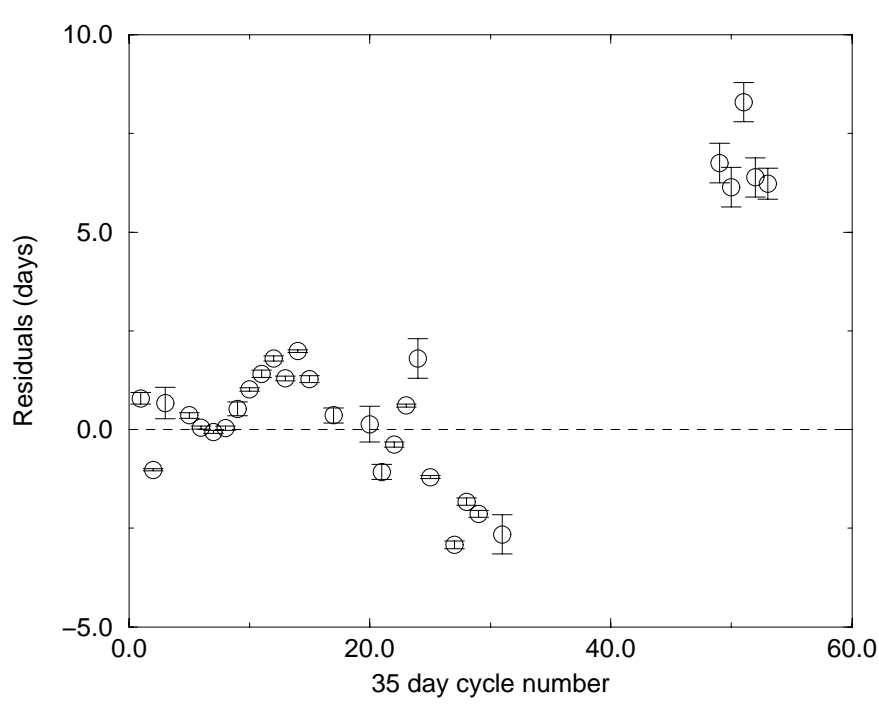

Fig. 5. The residuals $(\mathrm{O}-\mathrm{C})$ of the mean times of the 35 day on-state with respect to the best-fit linear ephemeris obtained from on-states before the start of the anomalous low-state.

it is proposed that the mean period is 20.5 times the orbital period, i.e., 34.85 day and that the observed on-states can fluctuate around this value by 0 or $\pm \frac{1}{2}$ orbital cycles. This simple ephemeris gives a reasonable description of the observed main-on times, while the extrapolation to the main-ons observed after the anomalous low-state by the RXTE-ASM show that these occurred $\sim 7$ days (or $\sim 0.2$ in phase) late (or alternatively, $\sim 0.8$ in phase early etc.). This ambiguity is present because the 35 day cycle count is not available during the anomalous low-state. Figure 5 shows the residuals with respect to the best-fit ephemeris assuming the former case. A clear difference between the on-state occurrence times before and after the anomalous low-state is evident.

\section{Discussion}

We have observed Her X-1 during the first main-on state following the 1999 March to 2000 September anomalous low-state. We find that the $0.1-30 \mathrm{keV}$ light curves, spectra, and pulse profiles are indistinguishable from those of previous main-on states and we therefore conclude that we observed Her X-1 during a normal main-on state.

The rapid spin-down of Her X-1 continued during at least part of the anomalous low-state, such that the pulse period is now at a similar value to $\sim 15$ years ago. This implies a spin-down rate which is a factor of $\sim 9$ times larger than the average spin-up rate. Vrtilek et al. (2001) noted that the magnitude of deviation from spin-down appears to be correlated with the length of the anomalous low state; this observation confirms this. There is no indication that the mass accretion rate changed substantially during the anomalous low-state since optical observations detected the effects of continued strong X-ray heating on the companion star (Margon et al. 1999). This implies that the spin-down torque originating from approximately the same amount of mass transfer was $\sim 9$ times stronger during the anomalous low-state than during normal intervals. Periods of more rapid spin-up than average are seen occasionally in Her X-1, but the magnitude of the spin-down rate observed during the recent anomalous low state is still larger by a factor of $\sim 2.5$ than the most rapid spin-up rate previously observed (around day 7300-8000 in Fig. 2).

This imposes constraints on any theory modeling the accretion torques in this source. The low-mass X-ray binary 4U 1626-67 underwent a torque-reversal (accompanied by a change in the spectrum, see Chakrabarty et al. 1997), but with only a change in the sign (not magnitude). This appears substantially different then a torque-reversal combined with a change in magnitude of a factor $\sim 9$ observed here. It has been suggested ( $\mathrm{Li} \&$ Wickramasinghe 1998) that variations in the spin-down rate can be caused by changes in the structure of the outer magnetosphere of the neutron star. In our case these changes may be accompanied by changes in the disk structure which produce the anomalous low-state.

The measured mean times of the main-on states that occur after the anomalous low-state occur at times inconsistent by at least $\sim 7$ days, or 0.2 in 35 day phase, with a simple linear extrapolation of the previous on-state times. However, we cannot exclude that this is actually 0.8 cycles earlier in 35 day phase, or some other multiple of the cycle. This difference may be explained by either a change in the 35 day phase at which the main on-states occur, or by a change in the underlying 35 day recurrence interval. It is probable that seventeen 35 day cycles occurred during the anomalous low-state. A change of $\sim 1.5 \%$ in the 35 day period could give rise to the observed difference. As Ögelman (1987) points out, the largest (-6.5 day) 35 day phase excursion recorded previously occurred at the time of the 1983 anomalous lowstate. This suggests that the occurrence of the anomalous low-states and mechanism responsible for the 35 day cycle in Her X-1 are somehow linked. Shakura et al. (1998) report a positive correlation between the length of the 35 day cycle and the mean X-ray flux. During the anomalous lowstate the effects of strong X-ray heating on HZ Her were still evident (Margon et al. 1999). However, there is no reliable estimate of how much the mean X-ray flux changed compared to intervals when the 35 day cycle is present. Indeed, since the mechanism producing this correlation is unknown, it is unclear whether the amount of X-ray heating experienced by HZ Her is a reliable indicator for this effect. This correlation suggests that during the anomalous low-state whichever part of the Her X-1 system regulated this mechanism received a significantly different mean X-ray intensity than during normal intervals.

Acknowledgements. The BeppoSAX satellite is a joint Italian and Dutch programme. We thank the staffs of the BeppoSAX Science Data and Operations Control Centers for help with these observations. We thank the RXTE instrument teams at MIT and NASA/GSFC for providing the All-Sky Monitor data. 


\section{References}

Boella, G., Chiappetti, L., Conti, G., et al. 1997, A\&AS, 122, 327

Chakrabarty, D., Bildsten, L., Grunsfeld, J. M., et al. 1997, ApJ, 474, 414

Coburn, W., Heindl, W. A., Wilms, J., et al. 2000, ApJ, 543, 351

Crosa, L., \& Boynton, P. E. 1980, ApJ, 235, 999

Dal Fiume, D., Orlandini, M., Cusumano, G., et al. 1998, A\&A, 329, L41

Deeter, J. E., Boynton, P. E., Miyamoto, S., et al. 1991, ApJ, 383,324

Delgado, A. J., Schmidt, H. U., \& Thomas, H. C. 1983, A\&A, 127, L15

Frontera, F., Costa, E., Dal Fiume, D., et al. 1997, A\&AS, 122, 371

Gerend, D., \& Boynton, P. E. 1976, ApJ, 209, 562

Giacconi, R., Gursky, H., Kellog, E., et al. 1973, ApJ, 184, 227

Jones, C. A., \& Forman, W. 1976, ApJ, 209, L131

Levine, A. M., \& Corbet, R. 1999, IAU Circ., 7139

Li, J., \& Wickramasinghe, D. T. 1998, MNRAS, 300, 1015

Margon, B., Deutsch, E. W., Leinhardt, Z. M., \& Anderson, S. F. 1999, IAU Circ., 7144

Mihara, T., \& Soong, Y. 1994, in Proc. of New Horizon of X-ray astronomy, ed. F. Makino (Universal Academy Press, Tokyo), 419
Ögelman, H. 1987, A\&A, 172, 79

Oosterbroek, T., Parmar, A. N., Martin, D. D. E., \& Lammers, U. 1997, A\&A, 327, 215

Oosterbroek, T., Parmar, A. N., Dal Fiume, D., et al. 2000, A\&A, 353, 575

Parmar, A. N., Pietsch, W., McKechnie, S., et al. 1985, Nature, 313, 119

Parmar, A. N., Martin, D. D. E., Bavdaz, M., et al. 1997, A\&AS, 122, 309

Parmar, A. N., Oosterbroek, T., Dal Fiume, D., et al. 1999, A\&A, 350, L5

Scott, D. M., \& Leahy, D. A. 1999, ApJ, 510, 974

Shakura, N. I., Ketsarsis, N. A., Prokhorov, M. E., \& Postnov, K. A. 1998, MNRAS, 300, 992

Staubert, R., Bezler, M., \& Kendziorra, E. 1983, A\&A, 117, 215

Tananbaum, H., Gursky, H., Kellog, E. M., et al. 1972, ApJ, 174, L143

Van Kerkwijk, M. H., Chakrabarti, D., Pringle, J. E., \& Wijers, R. A. M. J. 1998, ApJ, 499, L27

Vrtilek, S. D., Mihara, T., Primini, F. A., et al. 1994, ApJ, 436, L9

Vrtilek, S., Quintrell, H., Boroson, B., et al. 2001, ApJ, 549, 522 